\title{
Misshandlung im Kindesalter Bleibende „Narben“ im Gehirn
}

Fragestellung: Kommt es als Folge von Misshandlungserfahrungen in der Kindheit zu strukturellen und funktionellen Veränderungen im Gehirn?

Hintergrund: Missbrauch und Misshandlung in der Kindheit stellen schwerwiegende Risikofaktoren für die Entstehung psychischer Störungen dar. Rund 30\% aller betroffenen Kinder erfüllen im späteren Leben die Diagnosekriterien einer depressiven Störung. Auch das Risiko für die Entstehung einer posttraumatischen Belastungsstörung (PTBS) steigt nach frühen Missbrauchserfahrungen signifikant an. Durch neurobiologische Untersuchungen konnte gezeigt werden, dass sich traumatische Erfahrungen in der Kindheit auch auf neurobiologischer Ebene niederschlagen können. So sind Missbrauchs- und Misshandlungserfahrungen mit einer Hyperreaktivität der Stressachse (Hypothalamus-Hypophysen-Nebennierenrinden-Achse) sowie mit Volumenminderungen bestimmter Hirnareale (u.a. der Hippocampusformation) assoziiert. Ähnliche neurobiologische Auffälligkeiten wurden auch bei Patienten mit Depression und PTBS beschrieben. Zudem zeigte sich bei diesen Patienten eine Übererregbarkeit der Amygdala - einer Struktur im limbischen System, die maßgeblich an der Verarbeitung von Emotionen beteiligt ist. Neurobiologische Veränderungen als Folge von Kindesmisshandlung könnten somit einen möglichen Risikomarker für später auftretende psychische Störungen darstellen.

Patienten und Methodik: 148 gesunde Probanden wurden in die Studie eingeschlossen und umfassend auf vergangene oder gegenwärtige psychische Störungen gescreent. Das Vorliegen von Kindheitstraumata wurde mithilfe des Childhood Trauma Questionnaire (CTQ) erfasst. Um die Reaktivität der Amygdala zu ermitteln, wurde das sogenannte „Emotional-Face-Matching-Paradigma" angewandt. Den Probanden werden hierbei Fotos von Menschen mit negativem/bedrohlichem Gesichtsausdruck präsentiert, während die Hirnaktivierung mithilfe funktioneller Magnetresonanztomografie (fMRT) gemessen wird. Auf struktureller Ebene wurde mit voxelbasierter Morphometrie das Volumen bestimmter Hirnareale untersucht.

Ergebnisse: Es bestand ein deutlicher Zusammenhang zwischen der Schwere der Traumatisierung in der Kindheit und strukturellen sowie funktionellen Veränderungen im Gehirn. Bei hohem Trauma-Score zeigte sich bei der Präsentation negativer Gesichter eine ausgeprägte Reaktivität der Amygdala (Abbildung 1). Diese Befunde wurden durch morphologische Messungen untermauert, in welchen Personen mit hohen CTQ-Werten Volumenminderungen unter anderem im Hippocampus aufwiesen.

Schlussfolgerungen: Belastende Erfahrungen in der Kindheit sind mit strukturellen und funktionellen Veränderungen im Gehirn assoziiert. Eine Hyperreaktivität der Amygdala sowie eine Volumenminderung des Hippocampus könnten ein Mediator zwischen Kindheitstraumata und späteren psychischen Störungen sein.
Dannlowski U, Stuhrmann A, Beutelmann $V$ et al. Limbic scars: Longterm consequences of childhood maltreatment revealed by functional and structural magnetic resonance imaging. Biol Psychiatry 2012; 71: 286-93
Kommentar: Dannlowski et al. zeigen eindrucksvoll, wie sich traumatische Kindheitserfahrungen auch bei psychisch gesunden Erwachsenen auf die regionale Hirnaktivierung und -struktur auswirken können. Die von den Autoren gefundenen „limbischen Narben" erinnern stark an die bei Patienten mit Depressionen oder Traumafolgestörungen beschriebenen Veränderungen. Die neurobiologischen Folgen soziobiografischer Risikofaktoren haben in der bildgebenden Hirnforschung bislang wenig Beachtung gefunden, insbesondere was die Untersuchung von psychisch Gesunden mit erhöhtem Erkrankungsrisiko anbelangt. Diese Arbeit trägt dazu bei, dass sich das ändert. Ein solcher Untersuchungsansatz ermöglicht einen vielversprechenden Brückenschlag zwischen epidemiologischer Risikoforschung und biologischer Psychiatrie, der dabei helfen kann, ätiopathogenetische Faktoren und - bei geeignetem Studiendesign - auch Resilienzfaktoren weiter zu erforschen. Es ist zu hoffen, dass dieser Untersuchungsansatz die psychiatrische Forschung in ähnlicher Form bereichern wird, wie das "Endophänotypen-Konzept", dass sich auf genetische Risikokonstellationen bezieht. Ob die individuelle Aussagekraft hier verwendeter oder aus diesem Ansatz abgeleiteter Untersuchungsmethoden eines Tages dazu ausreichen wird, Menschen mit erhöhtem Erkrankungsrisiko zu identifizieren und zum Beispiel einer geeigneten Frühintervention zuzuführen, bleibt allerdings abzuwarten.

Cornelia Schwarze und Arian Mobascher, Mainz 